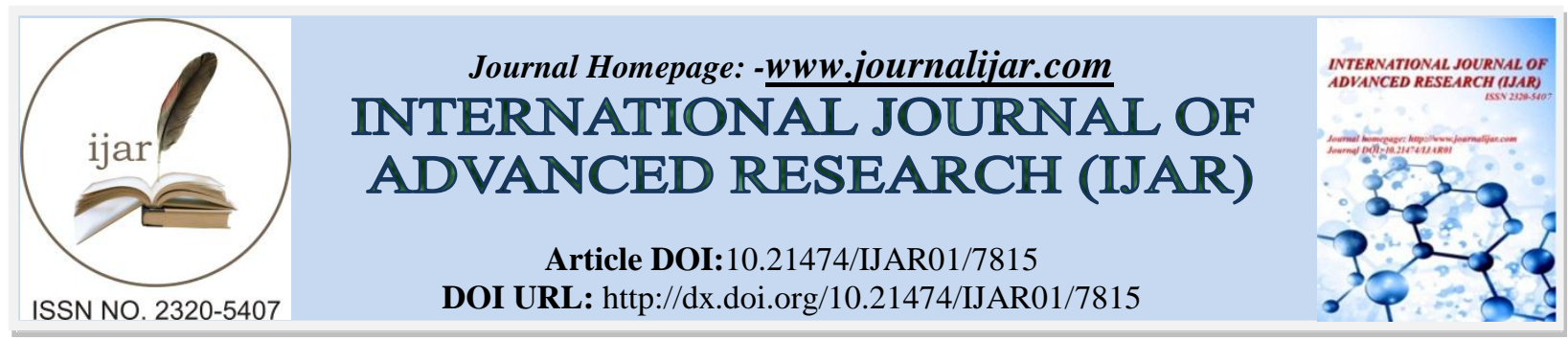

RESEARCH ARTICLE

\title{
COMPREHENSIVE TREATMENT OF CHRONIC WOUNDS OF VASCULAR ORIGIN.
}

E. Hadzhiev, V. Govedarski, P. Rana, T. Zahariev and G. Nachev.

Clinic for Vascular and Endovascular Surgery University Hospital "St. Ekaterina" Sofia, Bulgaria.

\section{Manuscript Info}

Manuscript History

Received: 6 August 2018

Final Accepted: 8 September 2018

Published: October 2018

Keywords:-

venous wounds, arterial wounds, mixed wounds.

\begin{abstract}
Introduction: The number of patients with chronic wounds of vascular origin is constantly rising due to the increased life expectancy of the population. $0.6-3 \%$ of the population aged 60 years and over, suffers from chronic wounds of the extremities. Untreated chronic wounds account for most amputations of the lower extremities.

Aim: This study aims at presenting the advantages of a comprehensive approach to treatment of chronic wounds of vascular origin based on our experience.

Materials and methods: This study comprises of 500 patients, which were treated for chronic wounds of vascular origin at the Clinic for Vascular and Endovascular Surgery at the University Hospital "St. Ekaterina" in Sofia and the "German Clinic" Medical Center in Gotze Delchev over a period of 5 years. Prior to their treatment, all patients underwent a complete vascular status assessment in order to determine the origin of their wounds: arterial, venous or mixed. 250 patients received only conservative treatment and 250 patients were assigned to the comprehensive treatment group.

Results: We assessed wound healing at 1, 3 and 6 months. Patients in the comprehensive treatment group showed a significant increase in wound healing as early as 1 month after beginning of treatment.

Conclusions: The comprehensive approach for treatment of chronic vascular wounds is of great importance. In order to select the appropriate method of treatment, the patients have to be examined by a skilled vascular surgeon or angiologist through a detailed assessment of the vascular system via Doppler examination and angiography.
\end{abstract}

Copy Right, IJAR, 2018, All rights reserved.

\section{Introduction:}

Increasing life expectancy of the population in recent decades already leads to an increase of elderly patients with vascular disease and patients with chronic wounds of vascular origin. Chronic wounds pose a significant problem not only in hospitals, but also in the everyday practice of general practitioners and physicians across many different specialties.[1] 0.6-3\% of the population aged 60 years and over suffer from chronic wounds of the extremities, and over 5\% of the aged 80 years and over are affected by this condition. Chronic arterial disease of the extremities is a frequent cause for wounds and varies between 1.9 and $13.1 \%$ across populations.[2] It is assumed that the frequency of chronic wounds increases because of an aging populations and accumulation of risk factors for atherosclerotic occlusion, such as stress, smoking, impaired metabolism, arterial hypertension, diabetes.[4, 3] Chronic arterial disease as a leading cause for chronic wounds of the extremities affects approx. 8 million American citizens and 12-

Corresponding Author: E. Hadzhiev. 
$20 \%$ of Americans between the age of 65 and 72 years.[5] According to a German study of leading causes for chronic wounds of vascular origin, chronic venous insufficiency is the leading factor with $47.6 \%$, arterial insufficiency causes $14.5 \%$ of wounds and $17.6 \%$ of ulcers are caused by a combined arterial and venous insufficiency. Rare causes include vasculitis (5.1\%), exogenous factors (3.8\%) and pyoderma gangrenosum (3.0\%).[6] According to other authors venous anomalies and conditions are amongst the most frequent health issues and about $70 \%$ of ulcers of the lower extremities are caused by venous disease.[3]

Fig. 1:-Causal distribution of chroming wounds

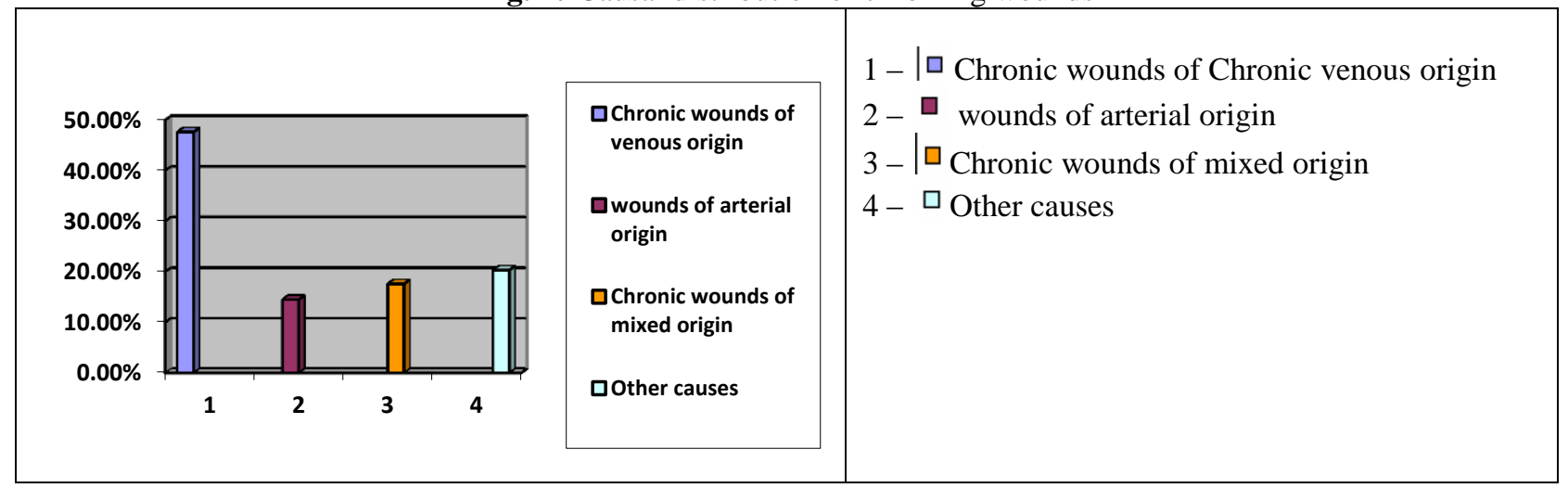

In Europe, 30\% of the adult population suffer from chronic venous insufficiency of the extremities [7,9] According to a study, published in 2006, $37 \%$ of patients who sought medical assistance in the Republic of Bulgaria suffer from chronic venous insufficiency of the extremities , $47 \%$ of the female and $30 \%$ of the male population.[8] During their lifetime women are 1.5 to 3.5 times as likely to suffer from chronic venous insufficiency as men.[8] Out of all patients with chronic venous insufficiency of the extremities , 1-2\% develop venous leg ulcers and approx. 300000 patients in the Republic of Bulgaria reach class V or VI according to the CEAP (Clinical, Etiological, Anatomic, Pathophysiological) Classification.[1,6] 10\% of patients who had a diagnosis of deep vein thrombosis (DVT) develop a chronic venous leg ulcer throughout their lifetime. [3,9]

A Chronic Wound Is a wound that heals by second intention and shows no tendency towards closure after 8 weeks, in spite of correct local treatment oriented towards the underlying causes.

Fig. 2:-2-month old mixed origin chronic wound

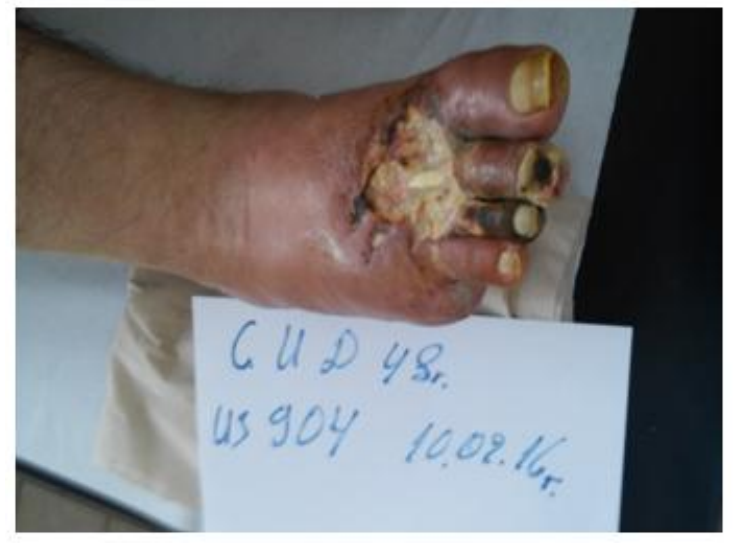

Chronic wounds of vascular origin are amongst the most common complications of impaired circulation in the lower extremities and diabetes. Impaired circulation in the lower extremities can be caused by obliterating processes of the aorta itself, as well as obliteration of peripheral arteries. All damage to blood vessels, even stemming from different 
causes, leads to impaired tissue perfusion with increasing ischemia and hypoxia, leading in turn to cell death and the formation of arterial trophic ulcers. [10]

$5 \%$ of the European population under 50 years of age suffers from peripheral arterial disease (PAD), $7 \%$ in the age group 50-60 years, $10 \%$ in the age group 60-70 years and $25 \%$ of everyone over the age of 70 .

Fig:-Epidemiology of PAD

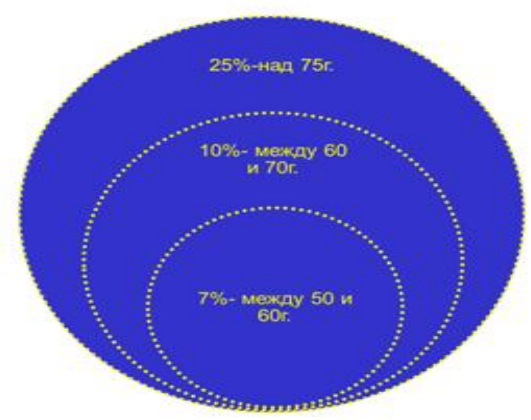

Men are 5 times as likely as women to suffer from PAD, but the gap narrows with increasing age. It is considered that $15 \%$ of all patients with diabetes develop a chronic wound at some point in their lives. For example, approx. $18 \%$ of all diabetic patients in the USA aged over 65 years have chronic non-healing leg wounds.[11] In 15-20\% of cases an amputation at different levels is required. Almost $85 \%$ of amputations are preceded by diabetic leg ulcers.

\section{Epidemiology of PAD}

Arterial trophic ulcers of the lower extremities are preceded by hypotrophic skin. Arterial ulcers are usually located on the toes, on the dorsal and plantar surface of the foot, and the heels and the bone protrusions of the foot. Ulcers appear with clear margins and a pale, non-granulating, necrotic base. [12] Chronic ulcers in patients, suffering from chronic venous disease (CVD) appear on swollen legs with subcutaneous varicose veins, dermo-hypodermitis, lipodermatosclerosis and oftentimes a previous episode of deep vein thrombosis (DVT).

The place of predilection is most commonly the outer or inner malleolus. [10]

Correct diagnosis is of utmost importance in order to avoid incorrect treatment that can lead to worsening of the condition, slowing of the healing process and potential harm to the patient. [13] Many patients with ulcers suffer for decades because of inadequate treatment attempts and the wrong approach to treatment on the part of physicians and patients.

Chronic ulcers of vascular origin remain a challenge for physicians from various specialties: vascular surgeons, surgeons, dermatologists, endocrinologists, rheumatologists and others.

Chronic wounds of vascular origin are treated conservatively and surgically, with medication, dressings, elevation of the affected extremity, vacuum therapy, compression bandages, ablation or endovenous procedures. $[1,2,14,15,16]$

\section{Aim:}

To present the advantages of a comprehensive approach to treatment of chronic wounds of vascular origin based on our experience.

\section{Materials and methods:}

For a period of 5 years 500 patients with chronic wounds of vascular origin have been treated at the Clinic for Vascular and Endovascular Surgery at the University hospital "St. Ekaterina" in Sofia; 300 of them had ulcers of venous origin, 120 patients had ulcers of arterial origin and 80 patients had wounds of mixed vascular origin. Patients were assigned to two treatment protocols. In the first group 250 patients received only conservative treatment. In the second group, 250 patients received comprehensive treatment, including surgery, wound dressings, vacuum therapy, elastic compression and medication. Conservative treatment included daily wound dressing, elevation of the afflicted extremity, multi-layer bandaging for approx. 14 days, and thereafter elastic compression stockings above the knee with $2^{\text {nd }}$ grade compression. 
Surgical procedures included stripping with ligation of incompetent perforating veins, radiofrequency ablation (RFA) of the great saphenous vein, surgical ulcer treatment and ligation of the perforating veins, endovascular stenting, dilation, Rotarex, bypass of different segments (iliac, femoral, and tibial blood vessels), profundoplasty.

The age distribution of our patients was as follows: 50 patients between 20 and 30 years of age, 60 patients between 30 and $40 \mathrm{y}, 100$ patients between 40 and $50 \mathrm{y}, 100$ patients between 50 and $60 \mathrm{y}$ and 120 patients older than 60 years, male/female ratio 320/200.

All patients underwent thorough diagnostic investigations before study start: complete vascular status assessment, Doppler ultrasonography, Doppler ultrasound and ABI Index, CT Angiography, phlebography at the physician's discretion, complete blood count, biochemical blood analysis, and microbiological samples from wounds/ulcers. Macroscopic wound evaluation: area, depth, necrosis, granulation, secretion and epithelialization.

\section{Inclusion criteria:}

Patients with chronic wounds of vascular origin, arterial or venous, irrespective of age and accompanying diseases.

\section{Results:}

We followed wound healing of chronic wounds of venous origin at 1, 3 and 6 months after start of treatment. Notably, there is no significant difference in wound healing between both treatment groups at the 3 -month mark.

The first group received conservative treatment: $55 \%$ healing rate at the 3 -month mark.

The second group received comprehensive treatment: $60 \%$ healing rate at the 3 -month mark.

However, there is a significant difference in relapse for a follow-up period of 1 year.

The conservative treatment arm shows a 30\% relapse rate, but the comprehensive treatment arm shows a relapse rate of only $15 \%$.

Unwanted events in the second group of patients, which underwent surgical treatment of the venous reflux were negligible.

\section{Comprehensive treatment of a chronic venous ulcer}

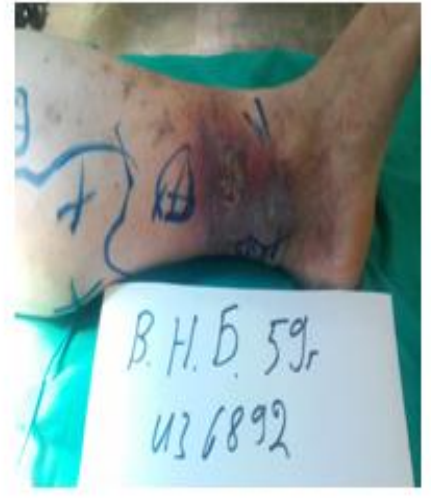

Fig. 3:-Day 1

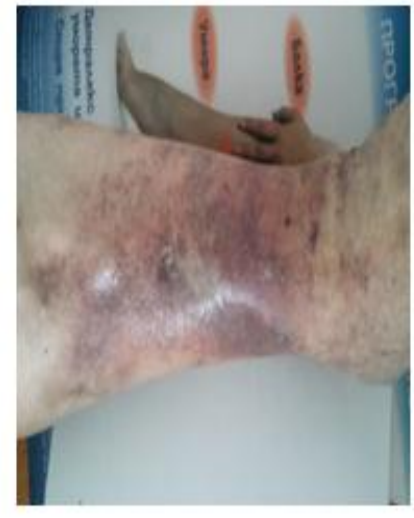

Fig. 4:-At 2 months

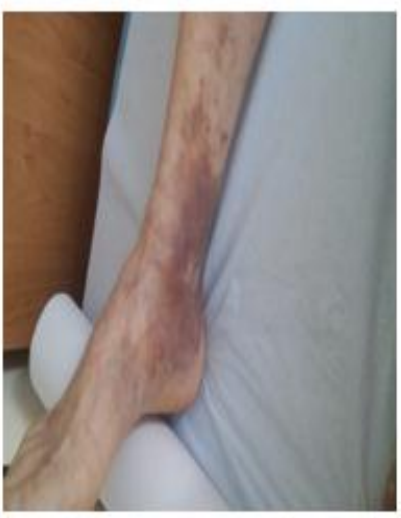

Fig. 5:-At 3 months 


\section{Comprehensive treatment of a chronic arterial ulcer (PTA, medication, surgical wound dressings, surgery)}

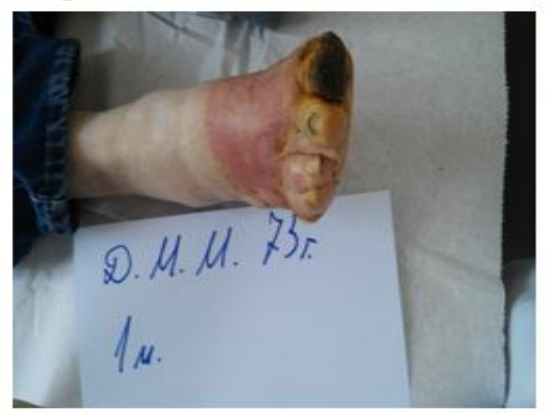

Fig. 6:- Day 1

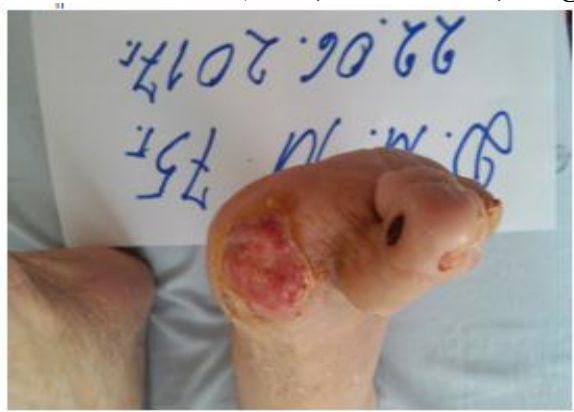

Fig. 7:-At 2 months

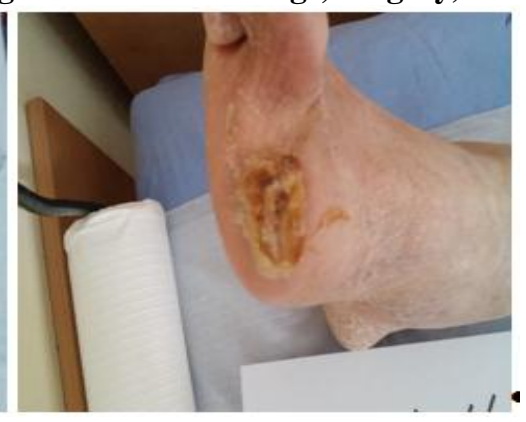

Fig. 8:-At 3 months

We monitored wound healing of chronic wounds of arterial origin an 1, 3 and 6 months after start of treatment. Patients were divided into 2 treatment arms.

The first group consisted of 250 patients, who received only conservative treatment, consisting of: medications (vasodilators, anticoagulants, antibiotics according to sensitivity testing, treatment of accompanying diseases, daily wound care with surgical and chemical debridement, wound dressings that support the healing process in all stages, vacuum therapy, etc.

The second group underwent comprehensive treatment and also consisted of 250 patients. Besides conservative treatment these patients underwent revascularization of the relevant vascular segment via different surgical techniques depending on the underlying cause, the occlusion level and accompanying diseases.

There is a notable difference in wound healing as early as 1 month after the beginning of treatment.

In the first (conservative) treatment arm 50 patients experienced a reduction of the wound area and depth by $20 \%$, fresh granulation tissue appeared and necrosis disappeared already at the 1-month mark. 25 patients showed complete wound healing. 20 patients underwent amputations: 10 at foot level, 6 at lower leg level and 4 at thigh level. At the 3-month mark 50 patients showed reduction of the wound area and depth by $15 \%$, fresh granulation tissue and partial necrosis. 50 patients showed complete wound healing. 10 patients underwent amputations: 4 at thigh level, 2 at lower leg level and 4 at foot level. At the 6-month mark 45 patients showed complete wound healing, 105 patients showed reduction of the wound area and depth with fresh granulation tissue, but incomplete wound healing. 4 patients underwent amputations, 2 trans-metatarsal and 2 of the lower leg and thigh. Amputations were indicated mainly for wounds of arterial and mixed vascular origin.

In the second (comprehensive) treatment arm 95 patients experienced complete wound healing at the 1-month mark. 70 patients experienced significant reduction of the wound area and depth by $60 \%$; necrosis disappeared completely and fresh granulation tissue appeared. 8 patients had to undergo amputations because of advanced ischemia: 4 at toe level, 2 at lower leg level and 2 at thigh level. At the 3-month mark 110 patients experienced complete wound healing. 60 patients showed partial wound healing but significant improvement and a tendency towards complete recovery. 5 patients had to undergo metatarsal amputations due to advanced uncontrollable infection. At the 6-month mark 20 patients showed complete wound healing and 20 patients showed partial wound healing. 2 patients underwent amputations at thigh level due to arterial re-thrombosis. 


\section{Comprehensive treatment}

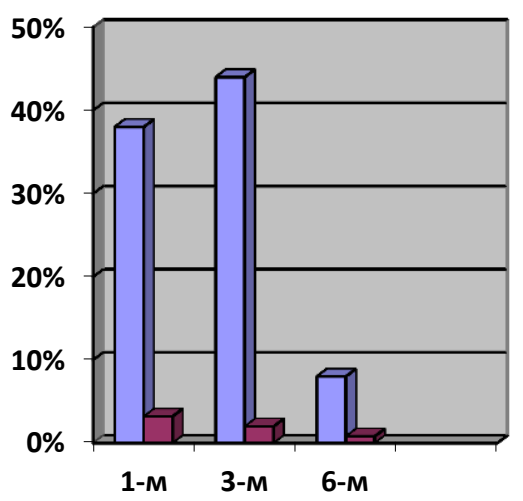

\section{Conservative treatment}
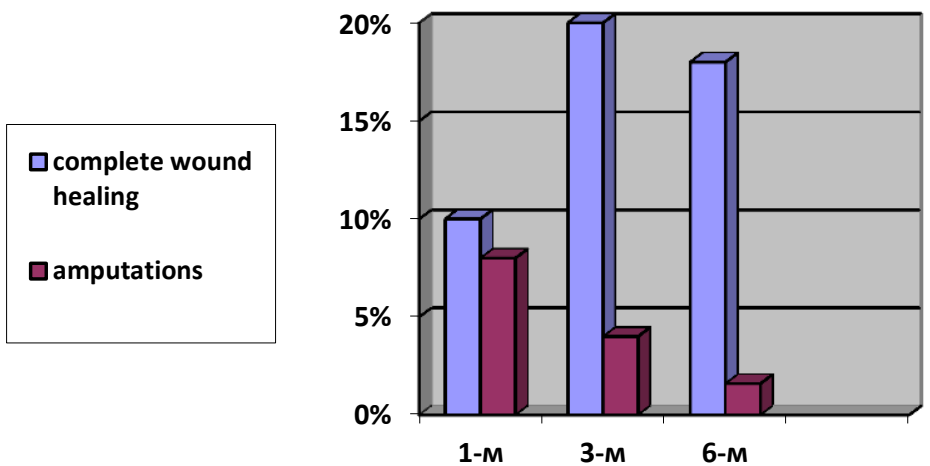

$\square$ complete wound healing

口amputations

Treatment and amputations at 1, 3 and 6 months

\section{Conclusions:}

1. Assessment of the vascular status and discovery of the underlying condition (impaired blood supply, venous reflux or both) is of utmost importance for patients with chronic wounds of the lower extremities.

2. Surgical and endovascular correction of the affected segments of the arterial and venous system is of utmost importance.

3. A comprehensive approach to treatment of chronic wounds of vascular origin is the treatment of choice.

4. Conservative treatment is only to be chosen if the potential for vascular reconstruction is exhausted.

5. Comprehensive treatment allows for shortening of treatment length and lowering of treatment costs and would significantly improve the quality of life of these patients.

\section{References:}

1. Birgit Kahle, PD Dr. med., ${ }^{,} 1$ Hans-Joachim Hermanns, Dr. med.,2 and Georg Gallenkemper, Dr. med.2 Evidence-Based Treatment of Chronic Leg Ulcers

2. C. Moffatt, "Leg ulcers," in Vascular Disease, S. Murray, Ed., pp. 200-237, Whurr Publishers, London, UK, 2001. View at Google Scholar

3. Jeroen D.D. Vuerstaek, MD, Tryfon Vainas, MD, PhDc, Jan Wuite, MDd, PattyNelemans, MD, PhDe, Martino H.A. Neumann, MD, PhDf, Joep C.J.M. Veraart, MD, PhD State-of-the-art treatment of chronic leg ulcers: A randomized controlled trial comparing vacuum-assisted closure (V.A.C.) with modern wound dressings

4. C. S. Sasanka, "Venous ulcers of the lower limb: where do we stand?" Indian Journal of Plastic Surgery, vol. 45 , no. 2, pp. 266-274, 201

5. C. K. Sen, G. M. Gordillo, S. Roy et al., "Human skin wounds: a major and snowballing threat to public health and the economy," Wound Repair and Regeneration, vol. 17, no. 6, pp. 763-771, 2009.

6. Körber, J. Klode, S. Al-Benna et al., "Etiology of chronic leg ulcers in 31, 619 patients in Germany analyzed by an expert survey University Clinic," Journal der Deutschen Dermatologischen Gesellschaft, vol. 9, no. 2, pp. $116-121,2011$.

7. Jantet G. RELIEF study: first consolidated European data. Angiology. 2000;51:31-37

8. Zahariev T. et al. Epidemiological study of chronic wasting disease in Bulgaria Phlebolymphology 2006

9. Nelson EA,Bell-Syer SE.; Compression for preventing recurrence of venous ulcers. Cochrane Database Syst Rev. 2012 Aug 15;8:CD002303. doi: 10.1002/14651858.CD002303.pub2

10. Zahariev T. Contemporary treatment of chronic wounds of vascular origin 2012 Sofia, Bulgaria.

11. Gones JE, Nelson EA. Skin grafting for venous leg ulcers. Cochrane Database Syst Rev. 2007;(2):CD001737

12. C. Moffatt, "Leg ulcers," in Vascular Disease, S. Murray, Ed., pp. 200-237, Whurr Publishers, London, UK, 2001. View at Google Scholar 
13. Marco AW Frade Department of Pathology, Grant Govt Medical College, Byculla, Mumbai 400008, India

14. R. Barwell, C.E. Davies, J. Deacon, K. Harvey, J. Minor, A. Sassano, et al.Comparison of surgery and compression with compression alone in chronic venous ulceration (ESCHAR study): randomised controlled trial

15. O.Medra S.,Cullum NA.,Nelson EA.; Compression for venous leg ulcers. Cochrane Database Syst Rev. 2009 Jan 21; (1):CD000265.

16. "Effect of elastic compression stockings in patients with varicose veins and healthy controls measured by strain gauge plethysmography" - M. Hirai, H. Iwata and N. Hayakawa, Dept. of Surgery, Aichi Prefectural College of Nursing and Dept. of Surgery, Toukai Hospital, Nagoya, Japan. 